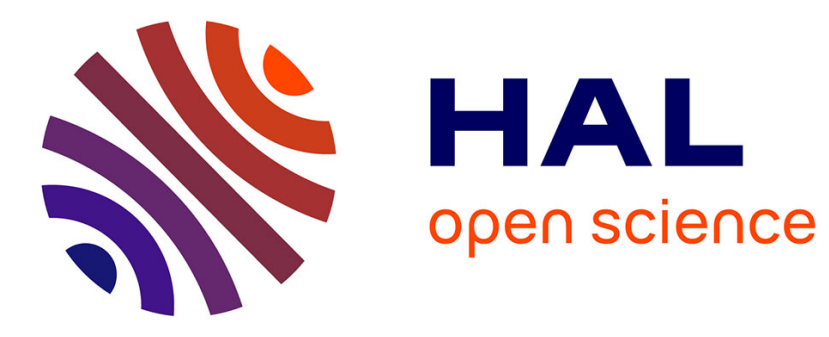

\title{
Lipomes cervicaux symptomatiques
}

\author{
Y. Najaf, C. Cartier, V. Favier, R. Garrel
}

\section{To cite this version:}

Y. Najaf, C. Cartier, V. Favier, R. Garrel. Lipomes cervicaux symptomatiques. Annales françaises d'Oto-rhino-laryngologie et de Pathologie Cervico-faciale, 2019, 136, pp.129 - 131. 10.1016/j.aforl.2018.05.007 . hal-03486474

\section{HAL Id: hal-03486474 \\ https://hal.science/hal-03486474}

Submitted on 20 Dec 2021

HAL is a multi-disciplinary open access archive for the deposit and dissemination of scientific research documents, whether they are published or not. The documents may come from teaching and research institutions in France or abroad, or from public or private research centers.
L'archive ouverte pluridisciplinaire HAL, est destinée au dépôt et à la diffusion de documents scientifiques de niveau recherche, publiés ou non, émanant des établissements d'enseignement et de recherche français ou étrangers, des laboratoires publics ou privés.

\section{다)(1) $(5$}

Distributed under a Creative Commons Attribution - NonCommercial| 4.0 International 


\section{Lipomes cervicaux symptomatiques}

Y. Najaf ${ }^{\text {a, }}$, C. Cartier ${ }^{\text {a }}$, V. Favier ${ }^{\text {a }}$, R. Garrel ${ }^{\text {a }}$.

${ }^{a}$ ORL et chirurgie cervico-faciale, Hôpital Gui de Chauliac, CHU de Montpellier, 34090 Montpellier, France

* Auteur correspondant : Yaser NAJAF : Interne d'ORL et chirurgie cervicofaciale, Montpellier, France. Email : Yaser.najaf@gmail.com 


\section{Résumé}

Introduction - Les lipomes sont des tumeurs bénignes fréquentes, que l'on retrouve dans l'ensemble du corps humain ; une minorité d'entre elles est située au niveau de la tête et du cou.

Résumé du cas clinique - Dans cet article, nous décrivons trois différents cas illustrés de lipomes cervicaux symptomatiques. Les diagnostics différentiels et les thérapeutiques y sont discutés à la lumière de la littérature médicale sur ce sujet.

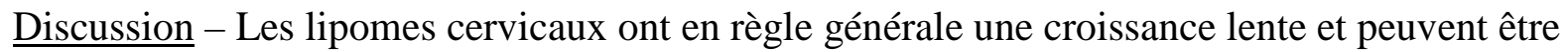
responsables de symptômes divers tels qu'une dysphagie, une dyspnée ou une dysphonie liées à un effet de masse, ou bien être uniquement révélés sur une gêne esthétique. Il est important de garder en mémoire le potentiel de transformation maligne de lipomes, qui représente un challenge diagnostique. Dans cet article, nous avons essayé de mettre en évidence la nécessité d'un dialogue entre chirurgiens, anatomopathologistes et radiologues pour le diagnostic et la prise en charge des lipomes de la tête et du cou.

Mots-clés: lipome, symptômes, imagerie. 


\section{Introduction}

Les lipomes sont des tumeurs bénignes d'origine mésenchymateuse, $13 \%$ d'entre elles sont situées dans la tête et le cou [1].

Ces tumeurs sont de consistance molle ou élastique, et de présentation clinique variable selon leur localisation, leur taille ou leur rythme de croissance. L'analyse anatomopathologique retrouve une tumeur organisée en lobules d'adipocytes matures. Les lipomes des voies aérodigestives supérieures sont souvent asymptomatiques. Néanmoins, ils peuvent être responsables de symptômes d'apparition lente telle qu'une dysphagie, une gêne cervicale, une dysphonie ou une dyspnée pouvant nécessiter une prise en charge en urgence [2]. Les examens d'imagerie doivent être réalisés devant toute masse cervicale indolore, et permettent d'éliminer la plupart des diagnostics différentiels. Dans cet article, nous décrivons 3 présentations cliniques révélées par des symptomatologies différentes et ayant donc bénéficié de prises en charge différentes.

\section{Cas 1}

Un homme de 80 ans ayant des antécédents cardiovasculaires présentait depuis un mois, présentait une symptomatologie à type de gêne à la déglutition et à la respiration associée à une toux chronique. Il ne se plaignait pas d'odynophagie, d'otalgie réflexe ni de perte de poids. La palpation cervicale ne révélait pas d'adénopathie. Un examen nasofibroscopique retrouvait un bombement du repli ary-épiglottique gauche faisant évoquer un laryngocèle. La mobilité laryngée était préservée. Un scanner cervical injecté (Figure 1) révélait la présence d'une masse pharyngo-laryngée supra-glottique pouvant correspondre à un laryngocèle mais dont la densité évoquait un tissu graisseux. Une résection endoscopique par laser $\mathrm{CO} 2$ a été réalisée, retrouvant une masse d'allure lipomateuse qui s'extériorisait à travers l'incision. La résection, dans ce contexte, a été partielle. L'examen anatomopathologique était compatible avec un lipome. Le patient a présenté une amélioration post-opératoire de ses symptômes et a ensuite bénéficié d'une rééducation orthophonique pour optimiser la récupération. Une imagerie post-opératoire montrait une réduction efficace du volume tumoral. Le suivi consistait en une visite annuelle pour le suivi des symptômes.

\section{Cas. 2}

Un homme de 49 ans présentait une masse cervicale gauche responsable d'une dysphagie d'apparition progressive. Il n'y avait ni dyspnée ni dysphonie. L'examen clinique ne retrouvait qu'une masse cervicale lisse, ferme, s'étendant jusqu'en arrière de la fourchette sternale. L'IRM retrouvait des arguments en faveur d'un lipome d'environ $10 \mathrm{~cm}$ de grand axe, situé entre l'artère carotide commune, la veine jugulaire interne et le nerf vague à gauche. Du fait de la 
symptomatologie, une exérèse chirurgicale a été réalisée, sous monitorage du nerf laryngé inférieur qui risquait d'être lésé. Une incision latéro-cervicale de $5 \mathrm{~cm}$ a été effectuée (figure 2). La résection était complète, sans complications neuro-vasculaire per ou post opératoire. Le patient signalait une disparition complète de la dysphagie deux semaines après la chirurgie.

\section{Cas 3.}

Un homme de 64 ans présentait un lipome cervical postérieur d'évolution progressive sur 20 ans. Une douleur nucale est apparue progressivement, sans aucun déficit neurologique. Cliniquement, (Figure 3), la masse était lisse, ferme et étendue en profondeur. Un scanner cervical montrait une masse lipomateuse de $13 \times 8 \mathrm{~cm}$ avec suspicion de transformation en liposarcome (calcifications multiples au centre de la masse adipeuse). Le patient a été opéré à visée symptomatologique et pour avoir une confirmation anatomopathologique. La masse pesait 450 grammes et correspondait à un lipome bénin. Les douleurs cervicales se sont progressivement amendées, mais la cicatrice, évoluant sur un mode hypertrophique, a nécessité des injections intralésionnelles de cortisone, avec un résultat esthétique satisfaisant.

\section{Discussion}

L'analyse de ces trois cas de lipomes cervicaux montre que ces tumeurs bénignes peuvent poser des difficultés lors du diagnostic initial, surtout quand il s'agit de masses cervicales symptomatiques. Ces masses peuvent engendrer uniquement une gêne esthétique, mais certains lipomes peuvent induire des signes de compression des voies aérodigestives supérieures ou être suspects de transformation maligne.

Les lipomes de la tête et du cou, bien que rares, sont souvent localisés dans le triangle cervical postérieur [3]. Nous présentons ici 3 cas de lipomes à développement antérieur : un lipome laryngé et deux masses cervicales étendues symptomatiques.

Le cas $n^{\circ} 1$ se présentait comme une masse pseudo-kystique supraglottique, ayant fait évoquer en premier lieu soit un laryngocèle, soit un kyste rétentionnel. Il s'agissait finalement d'un lipome laryngé développé aux dépends du ventricule. Epidémiologiquement, ces tumeurs ne représentent que $0,6 \%$ des tumeurs laryngées bénignes [4], provenant des zones graisseuses de l'épiglotte, du pli aryépiglottique ou du vestibule laryngé [5].

Les cas $\mathrm{n}^{\circ} 2$ et $\mathrm{n}^{\circ} 3$ illustrent l'importance des imageries préopératoires (échographie, scanner ou IRM) pour éliminer les diagnostics différentiels et guider la prise en charge chirurgicale. L'échographie a été utilisée en première intention pour explorer ces deux masses, mais ne permettait qu'une étude incomplète de leur extension. Classiquement, les lipomes sous des lésions hypoéchogènes homogènes [6] de caractéristiques variables en fonction de leur extension en profondeur et des tissus environnants [7].

L'analyse IRM de ces masses aide le chirurgien à visualiser le degré d'extension en profondeur, et les rapports vasculaires et nerveux avant l'intervention. Derin A. et al (2009) ont publié le cas 
d'un lipome cervical bénin infiltrant compliqué d'une rupture per-opératoire de l'artère carotide interne, ayant nécessité un pontage carotidien, ce qui illustre l'importance d'avoir une imagerie de qualité pour dépister d'éventuelles infiltrations lipomateuses périvasculaires [8].

De plus, l'imagerie joue un rôle important pour le dépistage d'éventuelles transformations malignes de ces lipomes. L'imagerie scannographique habituelle des lipomes bénins retrouve une masse hypodense homogène sans prise de contraste, alors qu' en IRM les signaux sont classiquement hyper-T1 avec une légère perte d'intensité en séquence T2 [9]. Il est admis que la majorité des lipomes ont une croissance lente, sont de volume limité et sont diagnostiqués par la clinique ; les lipomes géants de la tête et du cou $(>10 \mathrm{~cm})$ avec un taux de croissance élevé ont un potentiel de transformation maligne [10]. Le diagnostic différentiel des lipomes des cas $\mathrm{n}^{\circ} 2$ et $\mathrm{n}^{\circ} 3$, après exploration radiologique complète, était le liposarcome. Dans le cas $\mathrm{n}^{\circ} 3$, malgré une croissance lente, la taille tumorale dépassait les $13 \mathrm{~cm}$ et il existait des éléments radiologiques qui ont fait indiquer l'exérèse chirurgicale. Le Tableau 1 [11] illustre les facteurs statistiquement associés au liposarcome bien différencié en comparaison avec le lipome bénin, pouvant faire évoquer ce diagnostic dans le cas $n^{\circ} 3$.

\section{Conclusion:}

Dans la revue de la littérature, la stratégie chirurgicale des lipomes cervicaux est variable et doit être adaptée à la localisation et la taille de la masse, l'imagerie pré opératoire et l'expérience du chirurgien. L'âge et les antécédents du patient peuvent aussi orienter une éventuelle prise en charge chirurgicale en limitant au maximum la morbidité post-opératoire. Dans ce travail, nous avons essayé de présenter des cas inhabituels de lipomes cervicaux ayant nécessité une exérèse chirurgicale, sans pour autant oublier que la majorité de ces masses, asymptomatiques, sont simplement surveillées. L'exérèse complète de ces tumeurs est primordiale pour éviter la récidive et le suivi post opératoire doit être prolongé, en particulier pour les cas symptomatiques.

\section{Declaration d'intérêt}

Les auteurs déclarent n'avoir aucun conflit d'intérêt en rapport avec cet article

\section{Remerciements}

Nous souhaitons remercier les patients qui ont donné leur accord publication de ces cas. Ce travail n'a pas bénéficié de financement dédié.

\section{Consentements}

Les patients inclus dans cette étude ont donné leur consentement éclairé pour cette publication. 


\section{Références}

[1] Barnes L. (1985) Tumors and tumorlike lesions of the head and neck: In: Barnes L, ed. Surgical Pathology of the Head and Neck. New York, NY: Dekker. pp:747-758

[2] Persaud, R. (2002). A rare case of a pedunculated lipoma in the pharynx. Emergency Medicine Journal, 19(3), pp.275-275.

[3] Gourtsoyiannis, N. and Ros, P. (2005). Radiologic-Pathologic Correlations from Head to Toe. [New York]: Springer-Verlag Berlin Heidelberg.

[4] Kodiyan, J., Rudman, J., Rosow, D. and Thomas, G. (2015). Lipoma and liposarcoma of the larynx: case reports and literature review. American Journal of Otolaryngology, 36(4), pp.611615.

[5] Zbären, P., Läng, H. and Becker, M. (1995). Rare Benign Neoplasms of the Larynx: Rhabdomyoma and Lipoma. ORL, 57(6), pp.351-355.

[6] Korentager, R., Noyek, A., Chapnik, J., Steinhardt, M., Luk, S. and Cooter, N. (1998). Lipoma and liposarcoma of the parotid gland. The Laryngoscope, 98(9), pp.967-971.

[7] Paunipagar, B., Griffith, J., Rasalkar, D., Chow, L., Kumta, S. and Ahuja, A. (2010). Ultrasound features of deep-seated lipomas. Insights into Imaging, 1(3), pp.149-153.

[8] Derin, A., Güney, K., Turhan, M., Erdoğan, A. and Ağırdır, B. (2009). Giant cervical lipoma invading carotid artery: a case report. Kulak Burun Bogaz Ihtis Derg, 19(1) pp.28-31

[9] Salvatore, C., Antonio, B., Del Vecchio, W. Lanza, A., Tartaro, G. and Giuseppe, C. (2003). Giant infiltrating lipoma of the face: CT and MR imaging findings. American Society of Neuroradiology, 24. pp.283-286.

[10] Medina, C., Schneider, S., Mitra, A. and A Mitra, J. (2007). Giant submental lipoma: Case report and review of the literature. Plastic Surgery, 15(4).

[11] Kransdorf, M., Bancroft, L., Peterson, J., Murphey, M., Foster, W. and Temple, H. (2002). Imaging of Fatty Tumors: Distinction of Lipoma and Well-differentiated Liposarcoma. Radiology, 224(1), pp.99-104. 
Tableau 1. Facteurs faisant évoquer le diagnostic de liposarcome bien différencié en comparaison avec le lipome.

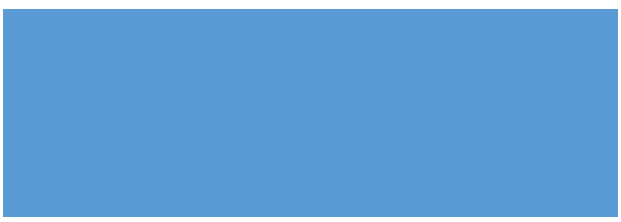

1. Sexe masculin

2.Age $>66$ ans

3. Faible \% de graisse au sein de la lésion $(<75 \%)$

4. Présence de calcifications

5. Masse $>10 \mathrm{~cm}$

6. Larges septations au sein de la lésion (>2mm)

7. Foyers nodulaires ou globuleux non lipomateux 
Figure 1: A gauche scanner pré-opératoire montrant un syndrome de masse supraglottique gauche développé aux dépends du reply aryépiglottique. A droite : Imagerie post opératoire montrant une réduction de volume et de l'effet e masse.

Figure 2 : A gauche : IRM pré-opératoire. A droite : Photographie per-opératoire. 1 : fourchette sternale, $2:$ lipome, $3:$ artère carotide commune, 4 . nerf vague, $5:$ veine jugulaire interne.

\section{Figure 3}

A gauche: Scanner cervical pré opératoire montrant le lipome cervical, étendu sur près de $180^{\circ}$. A droite : Photographie pré opératoire du patient 


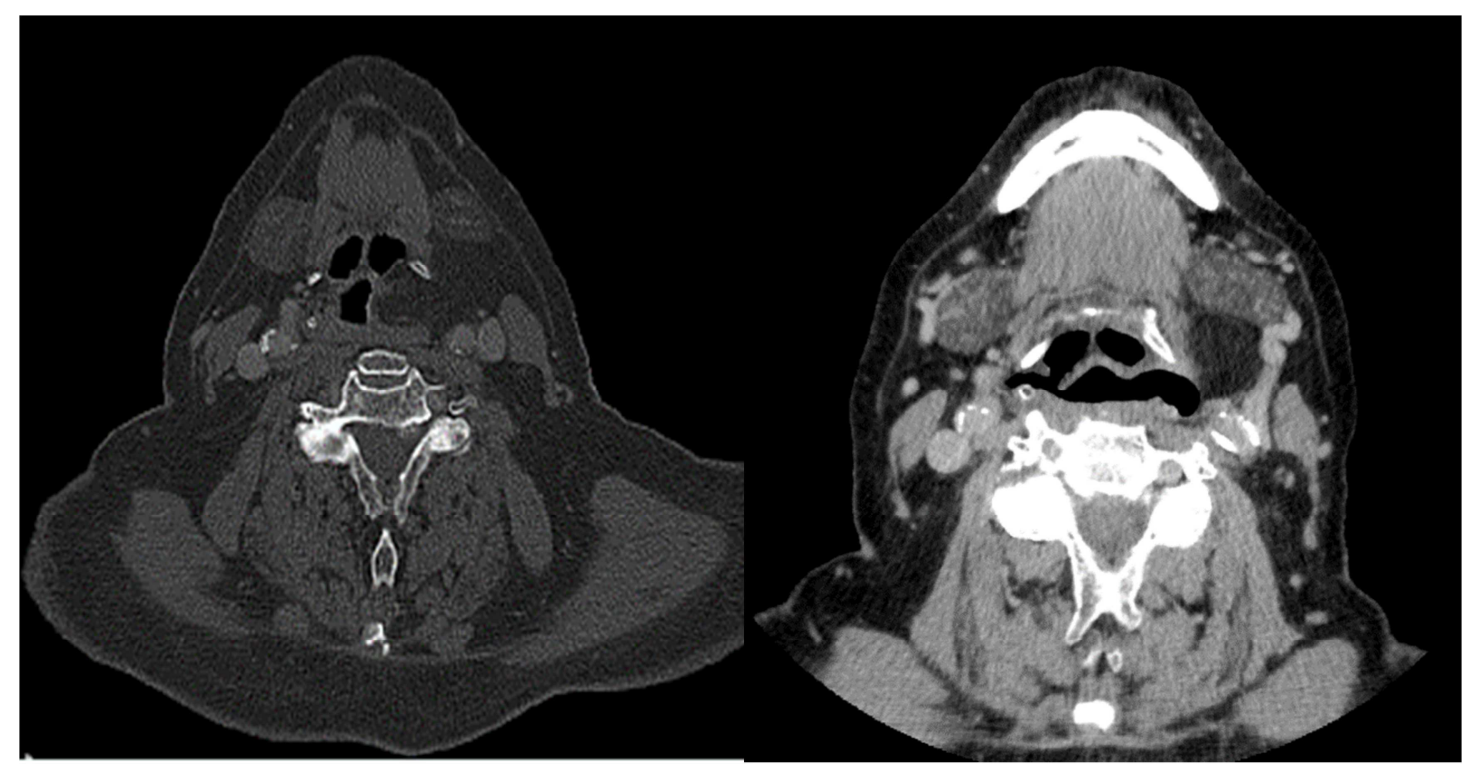

Figure 1 :

Figure 1 : A gauche scanner pré-opératoire montrant un syndrome de masse supraglottique gauche développé aux dépends du reply aryépiglottique. A droite : Imagerie post opératoire montrant une réduction de volume et de l'effet e masse. 


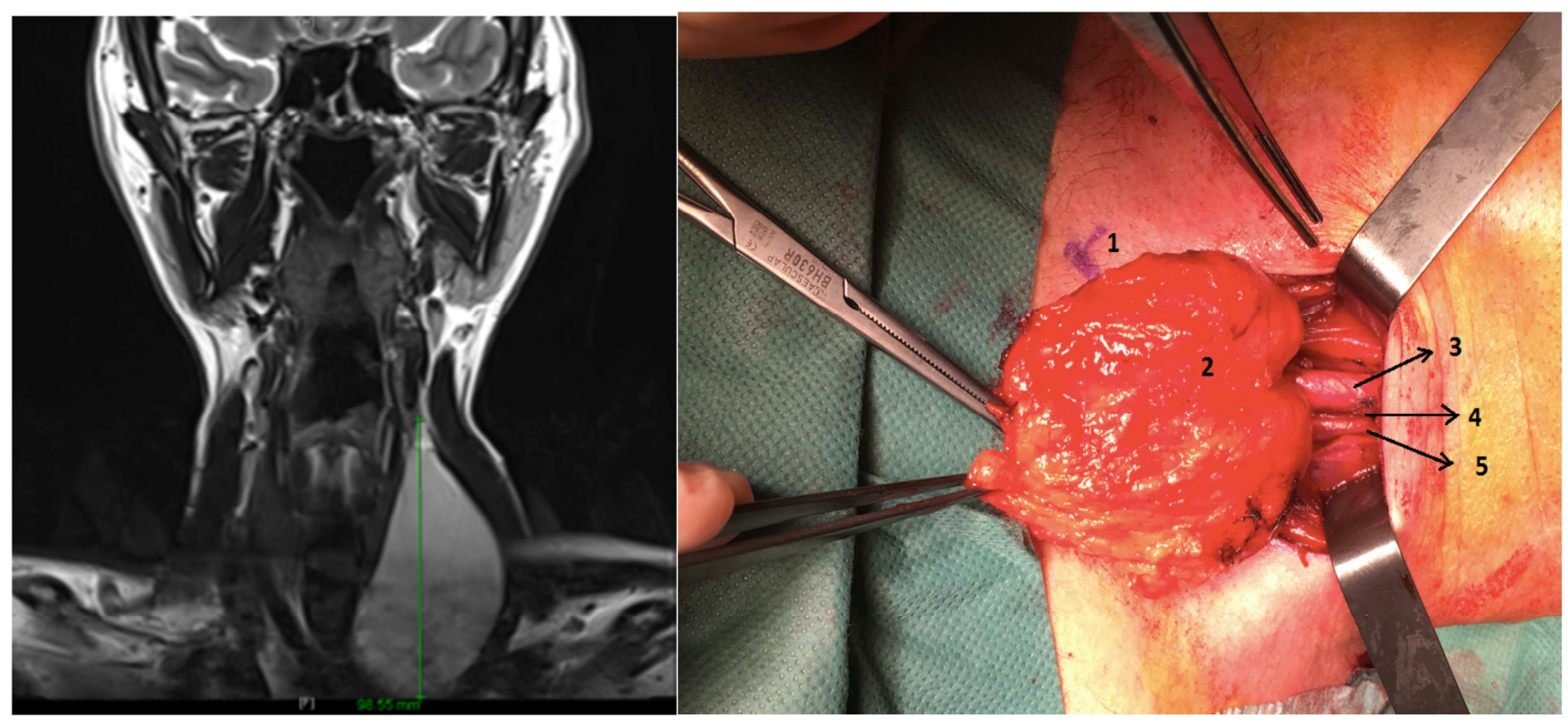

Figure 2 :

Figure 2 : A gauche: IRM pré-opératoire. A droite : Photographie per-opératoire. 1 : fourchette sternale, 2 : lipome, $3:$ artère carotide commune, 4 . nerf vague, $5:$ veine jugulaire interne. 


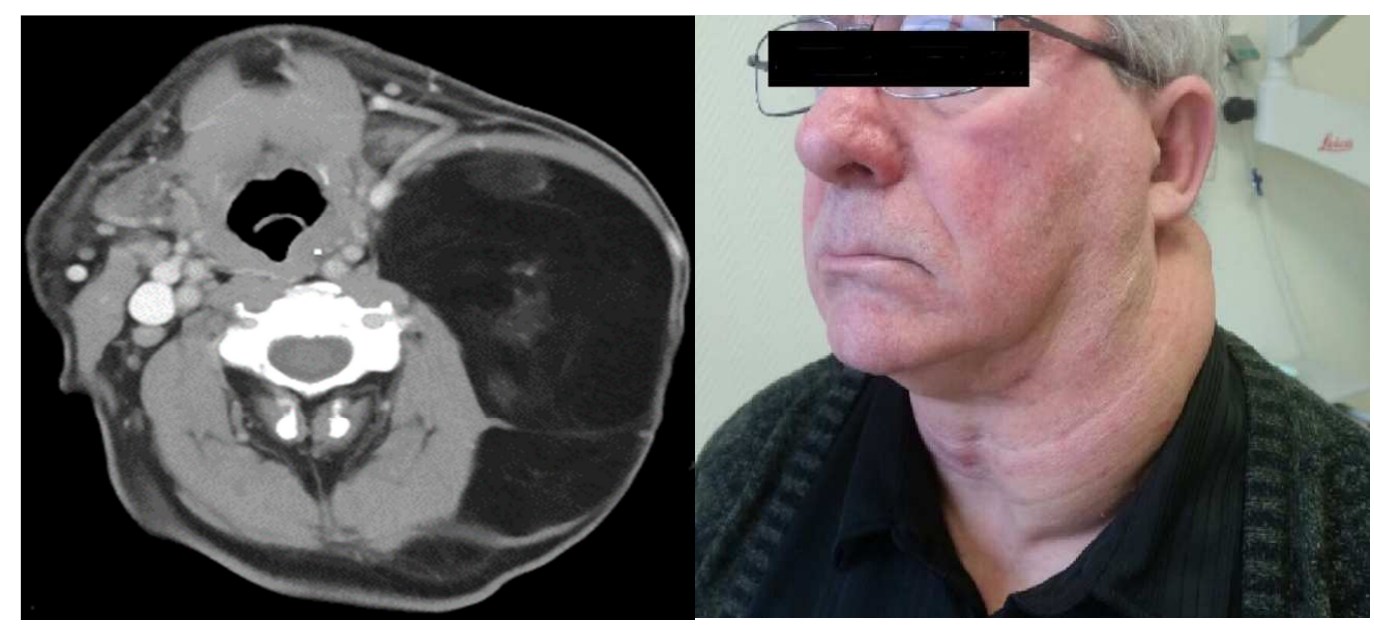

Figure 3

A gauche: Scanner cervical pré opératoire montrant le lipome cervical, étendu sur près de $180^{\circ}$. A droite: Photographie pré opératoire du patient 